\title{
Dynamic Equipment Workspace Generation for Improving Earthwork Safety Using Equipment Pose and State Data
}

\author{
Faridaddin Vahdatikhaki ${ }^{a}$ and Amin Hammad ${ }^{\mathrm{b}}$ \\ ${ }^{a}$ Department of Building, Civil and Environmental Engineering, Concordia University, Canada \\ ${ }^{b}$ Concordia Institute for Information Systems Engineering, Concordia University, Canada \\ E-mail: vahdatifarid@yahoo.com, hammad@ciise.concordia.ca
}

\begin{abstract}
In the period between 1992 and 2002, struck by vehicles and struck by objects (e.g., vehicle parts, vehicle loads, or falling vehicles) were identified as the causes of $30 \%$ and $24 \%$ of deaths on excavations sites, respectively. It is therefore of a paramount importance to improve the safety of construction sites by increasing the peripheral awareness of the operators of earthwork equipment. The existing collision avoidance systems often detect collisions based on the workspaces that only account for the geometry and the degrees of freedom of the equipment, and thus disregard the job-site-andstate-dependent characteristics of equipment. This results in reserving a large space for every piece of equipment. Therefore, this paper proposes a novel method for generating dynamic equipment workspaces based on the continuous monitoring of a spectrum of equipment-related information, i.e., the current pose/state of the equipment, and the speed characteristics of each movement. This method uses the required operation stoppage time to determine how much space needs to be reserved for each piece of equipment. A case study is conducted to validate the proposed method. It is shown that the proposed method has a strong potential in capturing the hazardous areas around the equipment and triggering warnings in view of the impending movements of various pieces of equipment.
\end{abstract}

Keywords - Dynamic Equipment Workspace, Earthwork Equipment, Real-time Location Systems

\section{Introduction}

With only less than $5 \%$ of the U.S. work force, the construction industry claims around $20 \%$ of fatalities and injuries in workplaces [1]. In the U.K., in addition to $25,000-30,000$ injured, approximately 1,500 people are losing their lives on construction sites in a typical decade [2]. Earthwork equipment accounts for a large share of injuries on construction sites. In the U.K., of the total number of fatalities in a period of 7 years (1996-2003), 14\% have been caused by being struck by a moving vehicle [3]. In the period between 1992 and 2002, struck by vehicles and struck by objects (e.g., vehicle parts, vehicle loads, or falling vehicles) were identified as the causes of $30 \%$ and $24 \%$ of deaths on excavations sites, respectively [4].

These statistics suggest that earthwork operations are in need of enhanced safety to avoid damages, injuries and fatalities. With this need in mind, many researchers have explored a wide range of solutions to mitigate the risk of accidents on excavation sites through reducing the possibility of collisions between equipment through a proper planning method $[5,6,7,8$, 9]. These methods identify the spaces required for the safe completion of different activities, i.e., activity workspaces, and try to reduce the overlap between them.

Despite the effectiveness of these methods in reducing the possibility of collisions between different teams of equipment at a macro level, they are not fully capable of averting safety risks emanating from human errors and unforeseen circumstances. Additionally, space is a limited resource that many earthwork projects do not have. These methods are not able to effectively improve the safety in congested sites, given that activity workspaces may overlap in many instances.

As a result, it is of a paramount importance to devise a complementary real-time mechanism to reduce safety risks based on the current pose and state of the equipment. Therefore, other researchers considered systems that generate warning against dangerous proximities based on the data of Real-time Location Systems (RTLSs) such as Global Positioning System (GPS), Radio Frequency Identification (RFID), and Ultra Wideband (UWB) $[10,11,12,13,14,15]$. These methods are applied at the monitoring phase aiming to ensure that different pieces of equipment do not collide with one another. Similar to the methods used at the planning phase, these methods consider the space around the equipment that should not be trespassed by other equipment to avoid potential collision in the immediate future. Because these spaces are applied to 
equipment, as opposed to the activities, and their shapes are dynamically changing based on the current pose of the equipment, they are referred to as Dynamic Equipment Workspaces (DEWs) in this paper. The correlation between the two types of workspace is that an activity workspace must be the envelope that contains all the DEWs generated by the fleet assigned to that activity over the scheduled period. Although DEWs are alternatively termed in the literature as "safety envelopes" $[10,15]$ or "safety zones" [16], the authors believe that, given the above correlation, it is preferable to use the unified term "workspaces" for both activity and equipment.

Two approaches can be found in the research addressing the generation of DEWs. One approach is to use only the equipment geometry and pose for the generation of DEWs [13, 14, 15, 17, 18, 31, 32, 33]. In this approach, the space around the equipment is overconservatively reserved within a radius (r) of the equipment (called here cylindrical workspace, Figure 1(a)). Or alternatively, the shortest distance between the two pieces of equipment is detected and a minimum acceptable threshold is used for generating the warnings, which is equivalent to considering only the pose of the equipment and creating a buffer of width $(b)$ around the equipment (called here buffer workspace, Figure 1(b)).

However, the cylindrical workspace reserves a large space for the safe performance of the equipment, considerably diminishing its effectiveness for the application in a congested site. On the other hand, while performing better in terms of economic use of space, the buffer workspace takes more time to detect potential collisions compared to the cylindrical workspace. In both cases, the shape of DEWs generated through these methods is determined by the Degrees of Freedom (DOFs) and the geometry of the equipment. Ignoring the movement characteristics of the equipment (i.e., the magnitude and direction of the instantaneous speed) results in reserving a large space around the equipment. However, a portion of this space can be safely used by other equipment if the workspace is defined more efficiently through considering the movement characteristics of the equipment.

Another approach is to use the information pertinent to the equipment movement characteristics to enhance the proximity measurement with a degree of prediction about the possible states of the equipment in the near future. These methods do not only rely on the proximity between various equipment and objects as the indication of imminent hazards, but also use the movement characteristics of the equipment to foresee if the equipment is likely to engage in potentially risky situations if it follows its current trajectory [10, 12, 16, 19, 20, 21, 22, 34]. However, these methods do not distinguish between different states of the equipment when generating the workspaces and do not cover the equipment with rotary movements, e.g., excavators. The valuable information about the state of the equipment can help better determine the size of the DEW in view of the potential dangers that may emanate not solely from the speed characteristics of the equipment but also from the nature of the equipment current state. Therefore, it could be argued that if the current pose, state and speed characteristics are better leveraged, it is possible to economize the space usage without sacrificing the effectiveness of the collision detection.

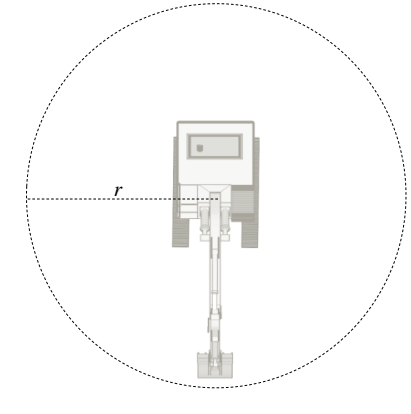

(a)

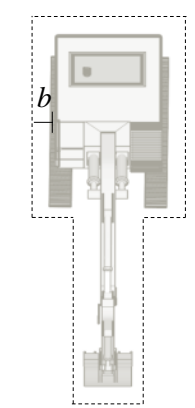

(b)
Figure 1. (a) Cylindrical Workspace, and (b) Buffer Workspace (the Model of Excavator is obtained from [23])

To the best of the authors' knowledge, the existing methods for generating DEWs do not take full advantage of the combination of valuable pose, state and speed characteristics of the equipment to accurately estimate the shape of DEWs. Consequently, the present research aims to leverage a set of information regarding the pose, state, and speed characteristics of the equipment to determine the shape and size of the workspace based on the required stoppage time of the equipment so as to secure the early identification of potential collisions while making a more economic use of space.

The structure of the paper is as follows. First, the previous relevant studies are presented. Then, the DEW generation method is elaborated. Next, a case study is elucidated as a means to validate the proposed method. Finally, the conclusions and future work are presented. This paper is a short version of a journal paper written by the authors [30].

\section{Proposed Method}

DEWs aim to use the pose, state, and speed characteristics of the equipment to generate a space around the equipment that would allow the prevention of immediate collisions with other pieces of equipment 
or obstacles on site, considering the equipment stoppage time $\left(t_{s}\right) . t_{s}$ can be used to determine how much of the space in the moving direction of equipment is unsafe after the operator becomes aware of a potential collision considering the operator reaction time and braking time. Although $t_{s}$ includes a period of moving with the current speed and a period of deceleration, in order to simplify the calculation process, this research conservatively assumes that the equipment continues to travel with its current speed and acceleration. Another assumption of the proposed method is that all pieces of equipment are equipped with an RTLS so that their poses and states can be calculated accurately. The update rate of DEWs is equal to the update rate of the RTLS used in the equipment. A rule-based system is used to identify the states of different equipment with a high accuracy by leveraging a set of equipment proximity and motion rules that determine the states of the equipment [24]. Also, a robust optimization-based method that uses geometric and operational characteristics of the equipment is used to improve the quality of the pose estimation [25]. Furthermore, in addition to the DEWs of equipment, semi-dynamic obstacles (such as trenches, temporary or permanent structures, etc.), also need to be represented by their own corresponding safety zones to enable effective collision avoidance at the global level.

In this research, excavators are used as an example for the explanation of the method since they have the most complicated movements in earthwork operations.

Figure 2 shows instances of a workspace that, unlike the DEWs shown in Figure 1, consider the pose, state, and speed characteristics of the equipment. In such a workspace, depending on the state of the excavator, different shapes are used. Figure 2(a) shows the DEW of an excavator in the swinging state that uses the magnitude of the rotation speed to determine the angle $(\alpha)$. However, ignoring the moving direction of the equipment may result in an uneconomic usage of the space that can be very valuable in a congested site. Therefore, it is recommended to consider the swinging direction of the equipment to differentiate between parts of the space to which the equipment is approaching and parts from which it is moving away. Figure 2(b) shows the proposed workspace of an excavator in the swinging state where the direction of rotation is considered to differentiate between the angles of the workspace on the rotation direction $(\alpha)$ and the opposite direction $(\beta)$. The rationale behind the asymmetric shape of the workspace is that the risk of collision along the direction of movement is much greater than along the opposite direction. Thus, a greater accent should be placed upon the space at the moving direction of the equipment. This research proposes asymmetric workspaces that consider the moving direction of a piece of equipment in each state as explained in the following sections. This arrangement better captures the potentially hazardous space around the equipment while using the space frugally, rendering this type of workspace very suitable for congested sites.

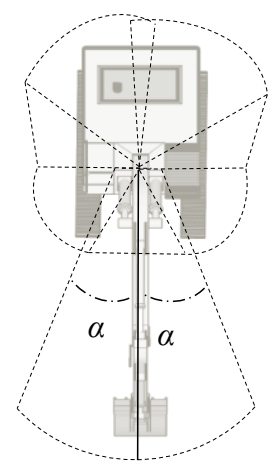

(a)

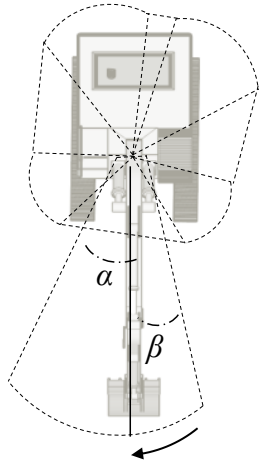

(b)
Figure 2. (a) Symmetric Workspace, and (b) Proposed Workspace

Figure 3 shows the flowchart for the generation of the proposed DEWs for excavators. With the 3D model of the equipment and its pose and state information available, the method proceeds to determine the linear and angular speeds of the equipment. For instance, an excavator can travel on its tracks with the linear speed of $\vec{v}$, move its bucket with the linear speed of $\overrightarrow{v_{b}}$, or swing with the angular speed of $\overrightarrow{\omega_{1}}$.

Upon the determination of the speed vectors, the DEW can be generated based on the type of the equipment and the equipment state as explained in the following sections. It should be emphasized that this method determines the shape of DEWs based on the assumption that the equipment is going to remain in its present state. Accordingly, the boundary situations, where the equipment is transiting between one state to another are not considered. However, this is tolerable in view of the high update rate of the DEWs. The types of the DEWs and the parameters that determine their shape are introduced in the following sections. Then, the potential collisions between workspaces are detected and warnings are sent to the involved operators.

While it is indispensable to account for workers onfoot in addition to the equipment and semi-dynamic structures for effective collision avoidance on a construction site, the current paper focuses only on the collision between equipment. This is because, given the size of the equipment and their inherently more complex kinematics, the interaction between equipment is more complex and more difficult to monitor. Nevertheless, the proposed method can be easily extended to consider the workspaces of workers as simple cylindrical shapes to avoid all types of collisions on a site.

Two distinct types of states can be identified for an 
excavator, namely stationary states (swinging, loading, dumping, and waiting) and traversal states (relocating, maneuvering). Usually an excavator can only engage in one of the two types at a time.

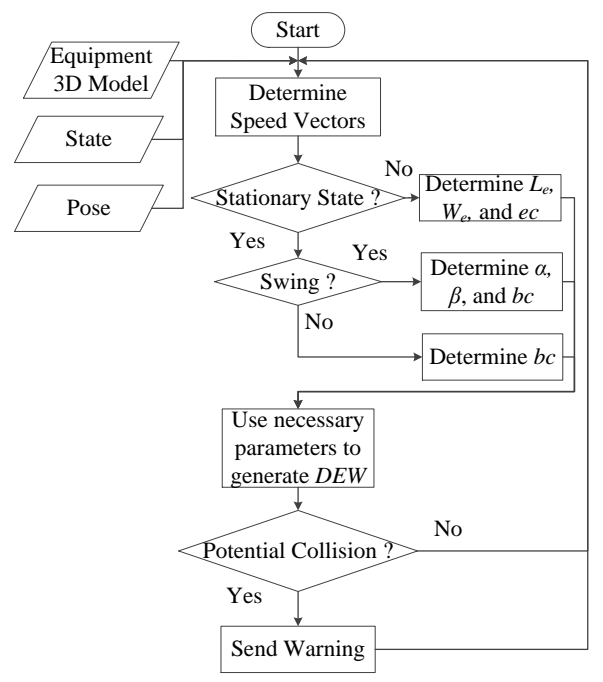

Figure 3. Flowchart of the Generation of DEW for Excavator

\subsection{DEW of Excavator in Stationary States}

As shown in Figure 4, a typical excavator can be controlled through five controllable DOFs resulting in the speed vectors $\overrightarrow{\omega_{1}}, \overrightarrow{\omega_{2}}, \overrightarrow{\omega_{3}}, \overrightarrow{\omega_{4}}$, and $\vec{v}$. However, since the workspace calculation is done in the $\mathrm{x}-\mathrm{y}$ plane, three of the above-mentioned DOFs $\left(\overrightarrow{\omega_{2}}, \overrightarrow{\omega_{3}}, \overrightarrow{\omega_{4}}\right)$ can be combined at any point in time to generate the instantaneous linear speed vector at the tip of the bucket $\left(\overrightarrow{v_{b}}\right)$. This reduces the number of the speed vectors to three $\left(\overrightarrow{\omega_{1}}, \vec{v}, \overrightarrow{v_{b}}\right)$.

When an excavator performs stationary operations, it only moves along either or both of $\overrightarrow{\omega_{1}}$ and $\overrightarrow{v_{b}}$. This is because a skillful operator is able to control multiple DOFs along $\overrightarrow{v_{b}}$ while swinging. The shape of the DEW is defined based on the identified current stationary state of the excavator (swinging, loading, dumping, and waiting). Additionally, since the tracks of the excavator are not moving during the stationary states, $D E W$ is defined only for the upper body of the excavator in these states.

\subsubsection{Excavator in swinging state}

As shown in Figure 5, if an excavator is identified to be in the swinging state with the angular speed of $\overrightarrow{\omega_{1}}$ and the linear speed of $\overrightarrow{v_{b}}$, the DEW is determined by the corresponding values of $\alpha, \beta$ and $\overrightarrow{v_{b_{x}}}$, where $\alpha$ represents the angle along the direction of rotation, $\beta$ represents the angle in the opposite direction that is reserved for the possible change of swinging direction instigated by unforeseen circumstances, and $\overrightarrow{\mathrm{v}_{\mathrm{b}_{\mathrm{x}}}}$ accounts for the combined movements of the boom, stick, and bucket in the vertical plane containing the axes of the boom and the stick. The DEW can be generated through the determination of the rotation radius for each bounding box $\left(R_{1}, R_{2}, R_{3}, R_{4}, R_{5}\right)$, a buffer $(b), t_{s}$, the rotation angles $\alpha$ and $\beta$, and the bucket motion clearance (bc), as shown in Figure 5(b).

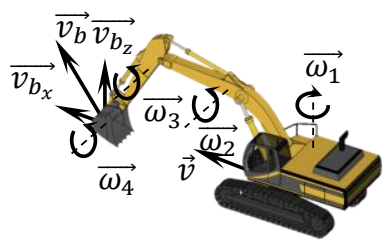

Figure 4. Speed Vectors Corresponding to Controllable DOFs for an Excavator (Model of the Excavator is obtained from [23])

$R_{l}$ is a variable that is defined as the distance from the excavator's center of rotation to the furthest point on the boom, stick, and bucket axis in the $\mathrm{x}-\mathrm{y}$ plane at the current time. $R_{2}$ to $R_{5}$ are fixed parameters that are dictated by the equipment geometry and correspond to the distances from the excavator's center of rotation to the front and rear corners of the upper body of the excavator. $b$ is also a fixed parameter used in order to define DEW with a degree of conservativeness. $b$ is proportionate to the size of equipment and can be defined as a percentage of the maximum dimension of the equipment, for example $1 \%$ of the length of the equipment, and is applied along the radii $R_{i}$. Other factors that may have impact on the value of $b$ are the update rate and the accuracy of the applied RTLS. Another buffer $(b)$ is added to the bounding box that contains the boom, stick and the bucket, as shown in Figure 5(b).

The angle $\beta$ represents the amount of swing the excavator will do over $t_{s}$ if the operator stops the swinging in its current direction and swings in the opposite direction for any reasons.

With this definition, $\beta$ is a function of $\omega_{1}, t_{s}$, and the swinging acceleration/deceleration $\left(\tau_{s}\right)$, assuming that they are equal. $\tau_{s}$ is a predefined value due to the fact that it pertains to the acceleration and deceleration that are expected to happen in case of swing direction shift. Equation (1) can be derived from basic kinematic equations [26] for the calculation of $\beta$.

$$
\beta=\frac{1}{2} \tau_{s}\left(t_{s}-\frac{\omega_{1}}{\tau_{s}}\right)^{2}-{\frac{\omega_{1}}{2 \tau_{s}}}^{2}
$$




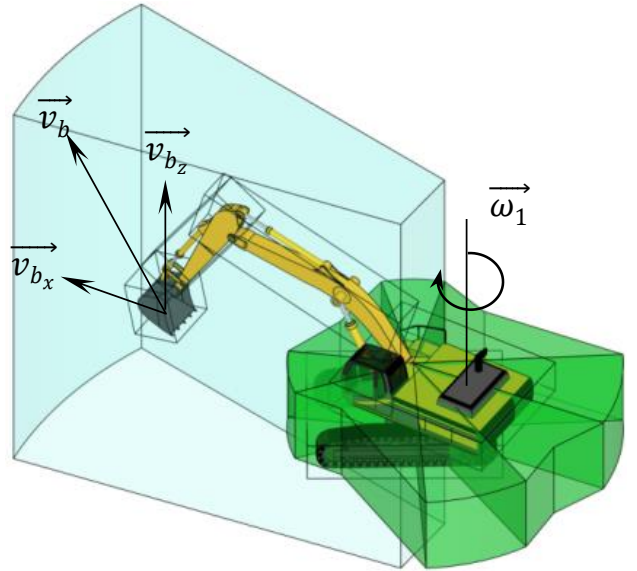

(a)

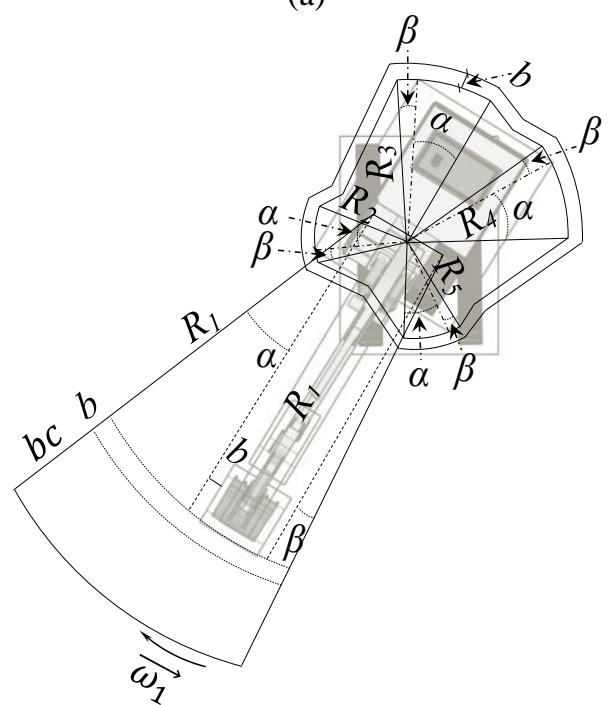

(b)

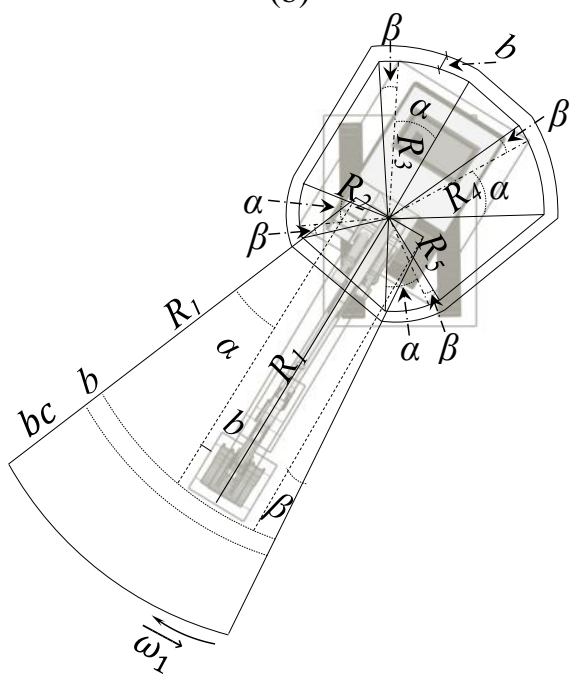

(c)

Figure 5. Schematic (a) 2D and (b) 3D and (c) simplified 2D Representations of DEW of an Excavator in Swinging State
The angle $\alpha$, on the other hand, denotes the amount of swing the excavator will be doing in the current direction provided it continues with its current speed $\left(\overrightarrow{\omega_{1}}\right)$ and acceleration/deceleration $\left(\tau_{a}\right)$. Unlike $\tau_{s}, \tau_{a}$ is a value measured in real time because it considers the current actual acceleration. Nevertheless, $\tau_{a}$ will be zero during most of the swinging operation since most excavators tend to reach to the steady state swinging speed quickly and then continue with that speed. Similarly, when the swinging is completed, the excavator decelerates to a complete halt quickly. Based on this definition, $\alpha$ is a function of $\tau_{s}, \omega_{1}$, and $t_{s}$, as shown in Equation (2).

$$
\alpha=\frac{1}{2} \tau_{\mathrm{a}} \times \mathrm{t}_{\mathrm{s}}^{2}+\omega_{1} \times \mathrm{t}_{\mathrm{s}}
$$

$b c$ represents the clearance buffer for the movement of the bucket along the boom, stick, and bucket axis when the skilled operator is combining the swinging motion with boom/stick/bucket movement away from the excavator [27]. It is determined by $\overrightarrow{v_{b_{x}}}$ (the projection of $\overrightarrow{v_{b}}$ on the horizontal plane), its corresponding measured acceleration $\left(\tau_{b_{x}}\right)$, and $t_{s \text {, as }}$ given in Equation (3). To generate $D E W$ conservatively, $b c$ is defined based only on the outward tilting of the combination of bucket/stick/boom movement and it ignores the inward tilting.

$$
b c=\frac{1}{2} \tau_{b_{x}} \times t_{s}^{2}+v_{b_{x}} \times t_{s}
$$

Although the accurate representation of the $D E W$ for the swinging state is as shown in Figures 5(a) and (b), a conservative simplification can be made to the geometry of the $D E W$ by connecting the corners of the pie shapes resulting from the rotations of each corner of the bounding boxes, as shown in Figure 5(c).

\subsubsection{Excavator in loading/dumping states}

Figure 6 shows the workspace when the excavator is in the loading/dumping states. Since the excavator's upper body is not swinging in these states, it is enough to reserve space for the movement of the boom/stick/bucket using a buffer. The shape presented in Figure 6 is the natural result of the excavator workspace in the swinging state, shown in Figure 5(c), when $\omega_{1}$ and $\tau_{s}$ are zero, and thus $\alpha$ and $\beta$ are zero. Accordingly, the workspace in these states is determined mainly by $b$ and $b c$, where the calculation of $b c$ is done similar to the case of the swinging state through Equation (3). 


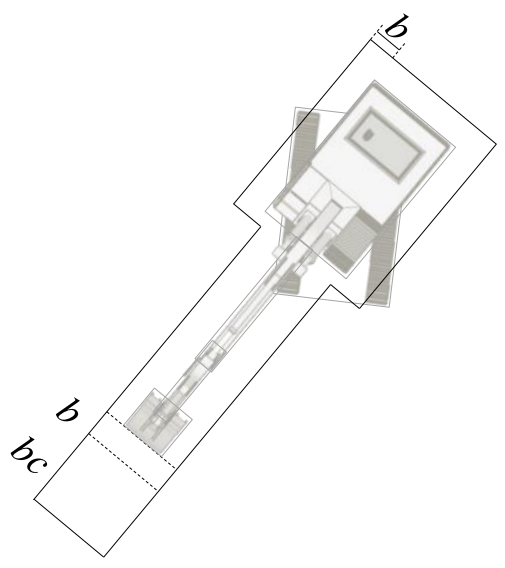

Figure 6. Schematic Representation of DEW of an Excavator in Loading and Dumping States

\subsection{DEW of Excavator in Traversal States}

Whereas Figures 5 and 6 illustrate the basic principle behind the generation of DEW for an excavator in stationary states, Figure 7 depicts the ruling parameters in forming the DEW when the excavator is performing traversal operations (i.e., relocating or maneuvering along $\vec{v}$ ). The workspace in this case is a box whose dimensions are regulated by (1) the dimensions of a bounding box representing the entire excavator $\left(L_{e}, W_{e}\right)$ at a given pose, where $L_{e}$ and $W_{e}$ are the instantaneous length and width of the equipment, a buffer $(b)$, and the excavator motion clearance $(e c)$. Unlike the workspace in stationary states, where the tracks were disregarded from the $\mathrm{DEW}$, in traversal states, the tracks need be incorporated in the workspace. This is because the tracks are not stationary and can be a source of collision risks. Given that the DEW is an instantaneous workspace generated solely based on the pose and the speed characteristics of the equipment, it is defined linearly along $\vec{v}$, even if the equipment is actually moving on a curved path. However, if the construction site has a road network, then the location data of the equipment can be integrated with the road data to ensure that the workspace is following the road alignment. This integration is not currently considered in this research but will be addressed in the future.

The rationale behind $b$ is similar to the one explained earlier in Section 1. ec represents the clearance buffer for the movement of the excavator when it moves on its tracks along the speed vector $\vec{v}$. It is therefore a function of $v$, measured $\tau_{t}$, and $t_{s}$ as given in Equation (4).

$$
e c=\frac{1}{2} \tau_{t} \times t_{s}^{2}+v \times t_{s}
$$

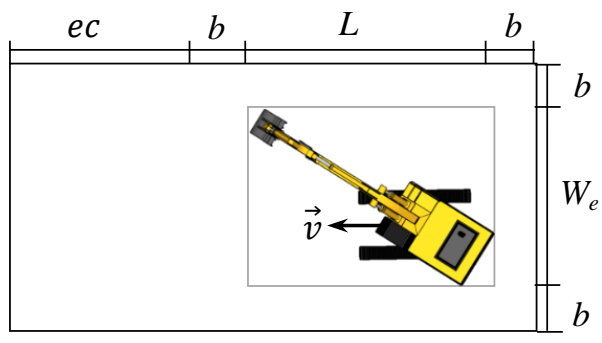

Figure 7. Schematic Representation of DEW of an Excavator in Traversal States

\section{Implementation and Case Study}

A case study was conducted to verify and validate the proposed method for generating DEWs. The data from a previously conducted lab test [24] were used to demonstrate the generation of DEW and its ability to effectively preempt potential collisions between equipment. As shown in Figure 8, three pieces of remotely controlled scaled equipment were employed to simulate an earthwork operation where an excavator is dumping a hypothetical load to a dump truck, which in turn hauls the material to a dumping zone, dumps it, and then returns to the loading spot for the next load.

Ubisense's Ultra Wideband (UWB) technology [28] was used to track the equipment. State and pose identification methods proposed in the authors' previous works [24, 25] have been deployed to provide the required input data. The update rate of UWB data after the pose correction method is $1 \mathrm{~Hz}$. The simulated operation consisted of four trips for the truck from the dumping zone to the loading zone, of which the first two loads were obtained from the stockpile 1 and the next two from the stockpile 2 .

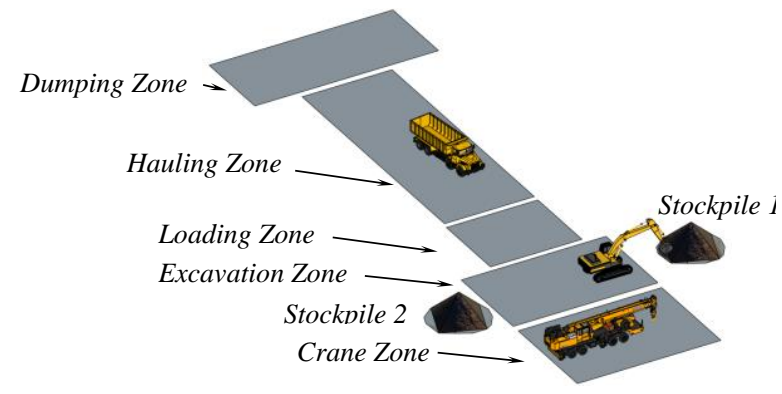

Figure 8. Schematic Layout of Simulated Site in the Case Study

With the intention to create a congested site, a crane was placed near the excavator without actively engaging in the simulated operation. Also, upon the completion of the fourth loading cycle, the excavator was intentionally 
steered to collide with the crane, to evaluate the effectiveness of DEW in preempting the potential collision.

The proposed method for the generation of DEWs was implemented using Microsoft Excel. The recorded UWB coordinates, the corrected pose, and the states of different pieces of equipment are imported into an Excel sheet as the input. The governing equations that generate DEWs were developed in Excel, as explained in Section 2. At every time step, the relevant speeds and accelerations/decelerations of the equipment are measured and the corresponding DEW is generated. An automated mechanism based on a variation of point in polygon algorithm [29] was also developed to identify the clashes between different DEWs.

In the generation of DEW, the values of $t_{s}, b$, and $\tau_{\mathrm{s}}$ were set to $2 \mathrm{~s}, 5 \mathrm{~cm}$, and $2 \frac{\mathrm{deg}}{\mathrm{s}^{2}}$, respectively. The values of $R_{2}, R_{3}, R_{4}$, and $R_{5}$ are set to $20 \mathrm{~cm}, 25 \mathrm{~cm}, 20$ $\mathrm{cm}$, and $25 \mathrm{~cm}$, respectively. Also, in order to enable complete collision detection, DEWs of truck and crane are considered similar to DEW of excavator in traversal state (Figure 7)

Figure 9 illustrates two snapshots of the generated DEWs at two stages of the simulated operation. The locations of attached tags on the equipment are indicated by the cross symbols. The DEWs of different pieces of equipment are shown using the dotted lines surrounding the equipment. The front of the truck is distinguished by the locations of the tags attached to the front of the bed.

Figure 9(a) shows a part of the operation when the truck was moving backward to adjust itself for loading. In this case, the extension of the DEW takes place at the rear of the equipment, representing the potential area of collision. Figure 9(b) shows the last phase of the operation where the excavator was intentionally steered towards a collision with the crane. As shown in this figure, the DEWs could be used to successfully identify and warning against the impending collision $4 \mathrm{~s}$ before the actual collision.

Based on the results of the case study, the proposed workspace occupied an average space of $0.25 \mathrm{~m}^{2}$. This represents $67 \%$ space savings when compared to a cylindrical workspace with the radius of $50 \mathrm{~cm}$. Defining the collision as any instances where the distance between the pair of equipment was less than 5 $\mathrm{cm}$, the proposed framework was able to warn against every collision within an average of $4.28 \mathrm{~s}$, which is only marginally less than the collision detection clearance time offered by cylindrical work space $(5 \mathrm{~s})$. In addition to significant space savings, the proposed workspace generated significantly less false alarms $(24.53 \%)$ compared to the cylindrical workspace $(68.81 \%)$

\section{Conclusions and Future work}

This paper proposed a novel method for the generation of real-time dynamic equipment workspaces considering the pose, state, and the speed characteristics of the equipment. This method is built on the previous work of the authors, where robust methods for the calculation of pose and state of different pieces of equipment based on RTLS data were presented. The present method considers the required operator stoppage time to determine how much space needs to be reserved in order to ensure that the equipment will not collide with other pieces of equipment in the immediate future. Excavators were used as the representatives of different types of equipment used in an earthwork project. The appropriate DEWs and their calculation process for all possible states of the equipment were presented.

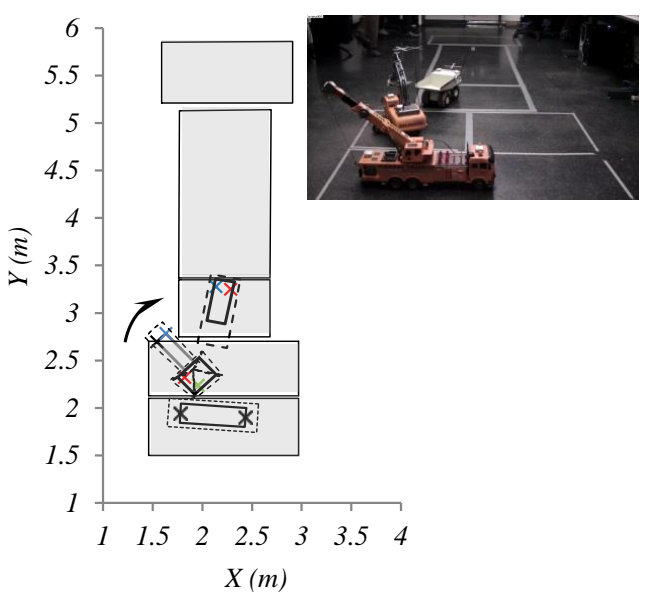

(a) backward motion of the truck

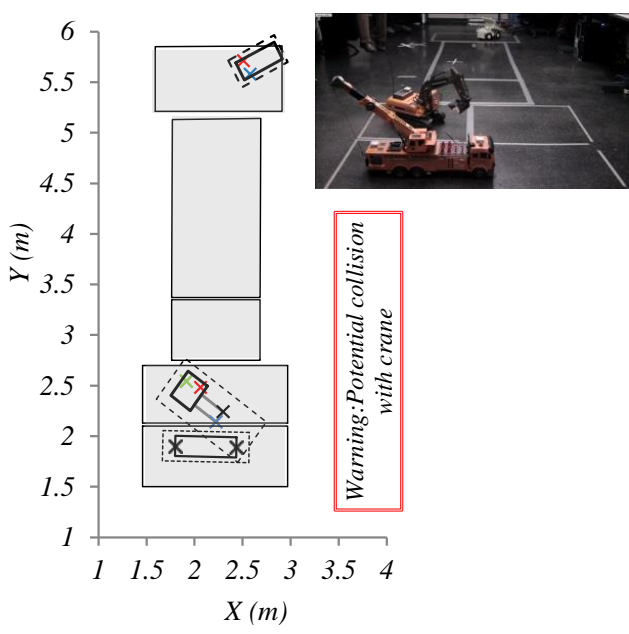

(b) Excavator's collision with the crane

Figure 9. Results of Generated DEWs of the Case Study 
In view of the results of the case study, it can be concluded that: (1) the proposed method is providing a balance between economic use of space and the ability to warn against potential collisions in an effective manner using the pose, state and speed characteristics of the equipment, (2) the flexibility of the method in using more than one speed vector in the calculation of DEWs enabled effective capturing of the operation of skilled operators where multiple DOFs can be used simultaneously.

Nevertheless, some false warnings resulted from capturing the movement along various DOFs only in 2D. Therefore, the future efforts of the authors are dedicated to avoiding this problem by considering the details of the movement in the third dimension in the generation of DEWs. Also, applying the proposed method in field tests is considered as future work.

\section{References}

[1] MacCollum D. V. Construction Safety Planning, John Wiley \& Sons, 1995.

[2] Davies V. J. and Tomasin K. Construction Safety Handbook, Thomas Telford, 1996.

[3] Howarth T. and Watson P. Construction Safety Management, Wiley-Blackwell, 2009.

[4] McCann M. Heavy Equipment and Truck-Related Deaths on Excavation Work Sites. Journal of Safety Research, 37(5):511-517, 2006.

[5] Chavada R., Dawood N. N. and Kassem M. Construction Workspace Management: The Development and Application of a Novel $\mathrm{nD}$ Planning Approach and Tool. ITcon, vol. 17, pp. 213-236, 2012.

[6] Mallasi Z. and Dawood N. Workspace Competition: Assignment, and Quantification Utilizing 4D Visualization Tools. In Proceeding of Conference on Construction Application of Virtual Reality, 2004.

[7] Tantisevi K. and Akinci B. Automated Generation of Workspace Requirements of Mobile Crane Operations to Support Conflict Detection. Automation in Construction, 16(3): 262-276, 2007.

[8] Hammad A., Zhang C., Al-Hussein M. and Cardinal G. Equipment Workspace Analysis in Infrastructure Projects. Canadian Journal of Civil Engineering, 34(10): 1247-1256, 2007.

[9] Moon H., Kim H., Kim C. and Kang L. Development of a Schedule-Workspace Interference Management System Simultaneously Considering the Overlap Level of Parallel
Schedules and Workspaces. Automation in Construction, 39: 93-105, 2013.

[10] Burns R. L. Dynamic Safety Envelope for Autonomous-Vehicle Collision Avoidance System. U.S. Patent, US 6393362, 2002.

[11] Carbonari A., Giretti A. and Naticchia B. A Proactive System for Real-Time Safety Management in Construction Sites. Automation in Construction, 20(6):686-698, 2011.

[12] Zhang C. and Hammad A. Improving Lifting Motion Planning And Re-Planning of Cranes With Consideration for Safety And Efficiency. Journal of Advance Engineering Informatics, 26(2), 2012.

[13] Guenther N. and Salow H. Collision Avoidance and Operator Guidance Innovating Mine Vehicle Safety. In Proceedings of the Queensland Mining Industry Health \& Safety Conference, Townsville, 2012.

[14] Wu H., Tao J., Li X., Chi X., Li H., Hua X., Yang R., Wang S. and Chen N. A Location Based Service Approach for Collision Warning Systems In Concrete Dam Construction. Safety Science, 51(1):338-346, 2013.

[15] Zolynski G., Schmidt D. and Berns K. Safety for an Autonomous Bucket Excavator during Typical Landscaping Tasks. New Trends in Medical and Service Robots, 20: 357-368, 2014.

[16] SAFEmine, Advanced Traffic Safety Solution for Surface Mining. Online: http://www.safemine.com/index.php/products\#cas. Accessed: 2014.

[17] Mining C. Shovel Load Assist Program. Online: http://www.crcmining.com.au/breakthroughsolutions/shovel-load-assist-project/. Accessed: 2014.

[18] Talmaki S. and Kamat V. R. Real-Time Hybrid Virtuality for Prevention of Excavation Related Utility Strikes. Journal of Computing in Civil Engineering, 28(3), 2014.

[19] Oloufa A., Ikeda M. and Oda H. GPS-Based Wireless Collision Detection of Construction Equipment. NIST special publication, 461-466, 2003.

[20] Worrall S. and Nebot E. A Probabilistic Method for Detecting Impending Vehicle Interactions. In Proceedings of IEEE International Conference on Robotics and Automation, Pasadena, CA, 2008.

[21] Hukkeri R. B. Machine Control System Implementing Intention Mapping. U.S. Patent US 
2012/0130582 Al, 2012.

[22] Wang J. and Razavi S. N. Low False Alarm Rate Model for Unsafe-Proximity Detection in Construction. Journal of Computing in Civil Engineering, 2015.

[23] Google 3D Warehouse, Online: https://3dwarehouse.sketchup.com/?redirect, Accessed:2015.

[24] Vahdatikhaki F. and Hammad A. Framework for Near Real-Time Simulation of Earthmoving Projects Using Location Tracking Technologies. Automation in Construction, 42:50-67, 2015.

[25] Vahdatikhaki F., Hammad A. and Siddiqui H. Optimization-based Excavator Pose Estimation Using Real-time Location Systems. Automation in Construction, (in press), 2015.

[26] Forshaw J. and Smith G. Dynamics and Relativity, John Wiley \& Sons, 2009.

[27] Rowe P. and Stentz A. Parameterized Scripts for Motion Planning. In Proceedings of the 1997 IEEE/RSJ International Conference on Intelligent Robots and Systems, 1997.

[28] Ubisense,Online: http://www.ubisense.net/en/, Accessed: 2015.

[29] Haines E. Point in Polygon Strategies. In Graphics gems IV, 24-26, 1994.

[30] Vahdatikhaki, F. and Hammad, A. Dynamic Equipment Workspace generation for improving earthwork safety using real-time location system. Advanced Engineering Informatics, (in press), 2015.

[31] Pradhananga, N. Construction site safety analysis for human-equipment interaction using spatiotemporal data." Doctoral dissertation, Georgia Institute of Technology, 2014.

[32] Marks, E. D., and Teizer, J. Method for testing proximity detection and alert technology for safe construction equipment operation." Construction Management and Economics, 31(6), 636-646, 2013.

[33] Teizer, J., Allread, B. S., Fullerton, C. E., and Hinze, J. Autonomous pro-active real-time construction worker and equipment operator proximity safety alert system." Automation in Construction, 19(5), 630-640, 2010.

[34] Cheng, T. Automated Safety Analysis of Construction Site Activities Using Spatiotemporal Data", Doctoral dissertation, Georgia Institute of Technology, 2013. 